\title{
A meta-review of meta-analyses and an updated meta-analysis on the efficacy of chloroquine and hydroxychloroquine in treating COVID19 infection
}

Nada Soliman1, Ahmed T. M. Aboughalia1, Tawanda Chivese ${ }^{1}$, Omran A. H. Musa ${ }^{1}$, George Hindy ${ }^{1}$, Noor Al-Wattary ${ }^{1}$, Saifeddin Badran ${ }^{1,2}$, Joshua T. Matizanadzo ${ }^{3}$, Mohamed M. Emara ${ }^{1}$, Lukman Thalib ${ }^{4}$, Suhail A. R. Doi ${ }^{1}$

${ }^{1}$ College of Medicine, QU Health, Qatar University, Doha, Qatar, ${ }^{2}$ Hamad General Hospital, Doha, Qatar, ${ }^{3}$ Brighton and Sussex Medical School, United Kingdom, ${ }^{4}$ Department of Public Health, QU Health, Qatar University, Doha, Qatar

\section{Background}

Controversy surrounds the efficacy of the antimalarial drug chloroquine (CQ), and its derivative, hydroxychloroquine (HCQ), on their efficacy and possible harm when used for the treatment of COVID19.

Findings from meta-analyses, and primary studies have produced conflicting findings on the efficacy and safety of these drugs.

\section{Aims \& Objectives}

To synthesize the findings from systematic reviews and meta-analyses as well as to update the evidence using a meta analysis in evaluating the efficacy and safety of CQ and HCQ with or without Azithromycin for the treatment of COVID19 infection.

\section{Methods}

A meta-review of published systematic reviews and updated meta-analysis of experimental studies where either HCQ or CQ with or without Azithromycin were used for the treatment of COVID19.

Search strategy:
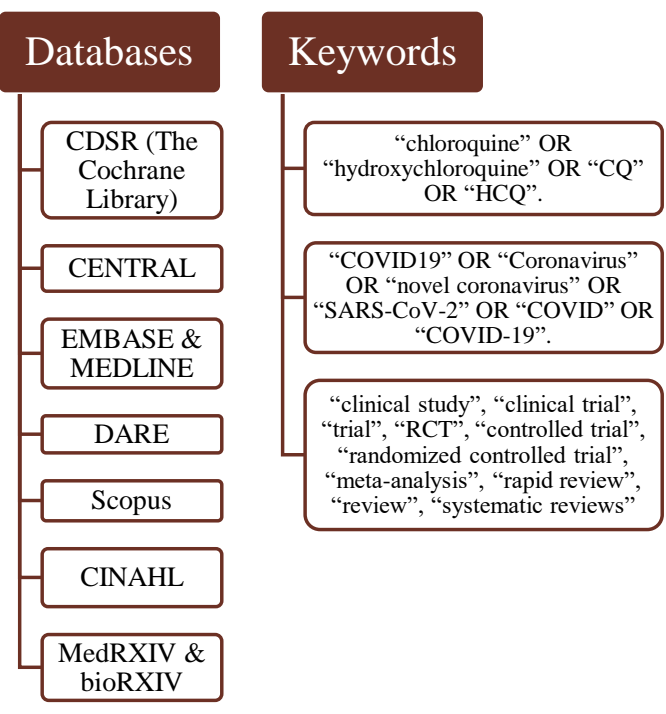

\section{Data extraction:}

For each study, two authors independently extracted data and assessed quality.

Quality Assessment:

Risk of bias was assessed using the Assessing the Methodological Quality of Systematic Reviews (AMSTAR) tool for reviews and the MethodologicAl STandard for Epidemiological Research (MASTER) scale for the experimental studies.

\section{Outcomes:}

- The main outcome for both the meta-review and the updated meta-analysis was mortality. Secondary outcomes were transfer to the intensive care unit (ICU) or mechanical ventilation, worsening of illness, viral clearance and the occurrence of adverse events.

\section{Synthesis of findings:}

Synthesis of findings from different reviews was done using a combination of a structured summary of findings from the reviews and presentation in forest plots.

For the updated met-analysis, findings from included experimental studies were synthesized using quality effect model.

The Cochran Q test p-value was used to test for and the $I^{2}$ statistic to quantify heterogeneity.
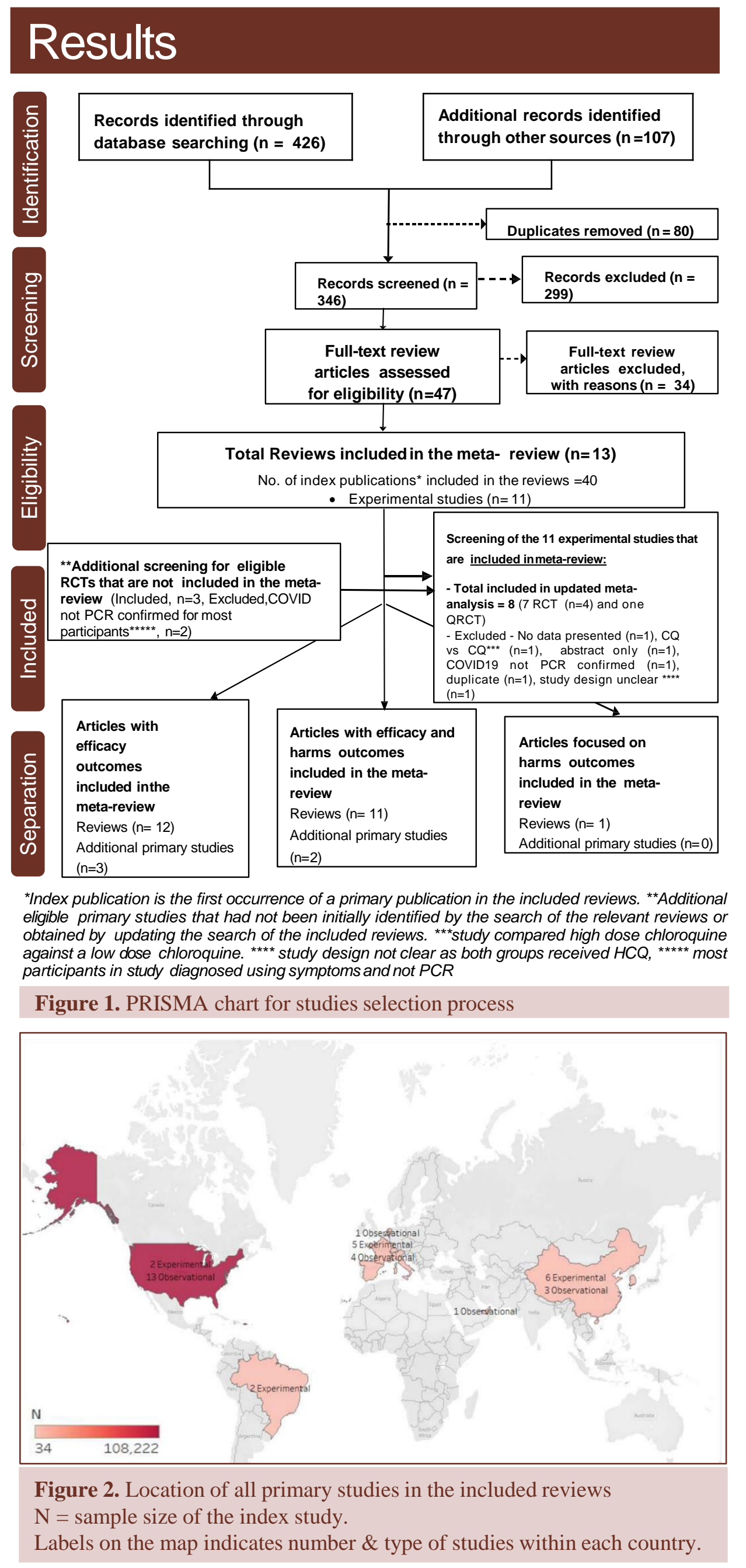

Meta-review of systematic reviews results All-cause of mortality

Overall, all meta-analyses showed higher risk of mortality with HCQ with/without Azithromycin, compared to control (Fig 3).

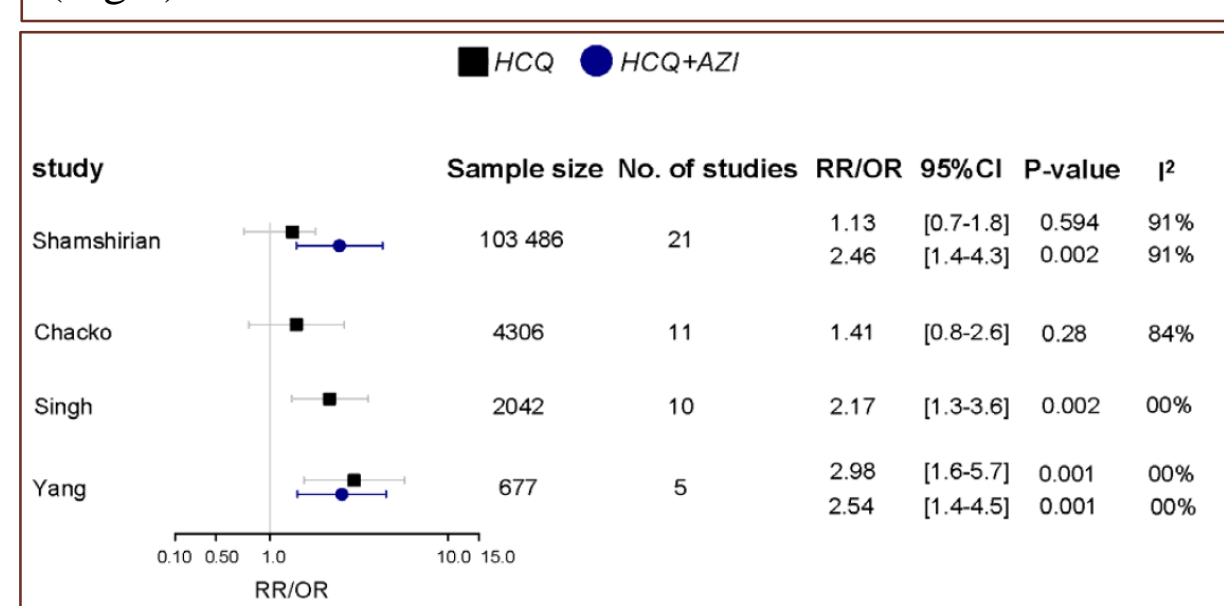

Figure 3. Results from meta-analyses included assessing all-cause of mortality outcome.

ICU transfer and disease exacerbation Most reviews found no significant differences between HCQ group and control, in the risk of transfer to ICU and disease exacerbation.

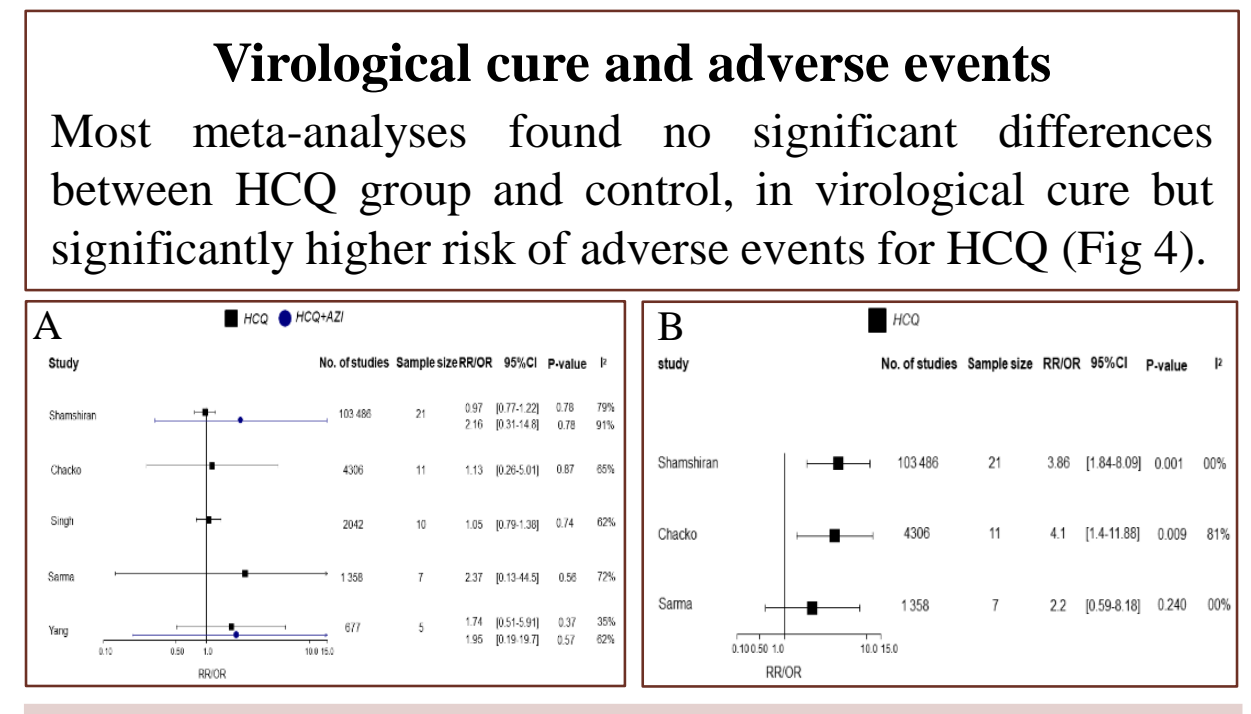

Figure 4. Results from meta-analyses included assessing A. virological cure and B. Adverse events.

Results of the updated meta-analysis of experimental studies

All-cause of mortality

There was no significant difference in risk of mortality between participants who received $\mathrm{HCQ}$ with or without Azithromycin and those on standard care (Fig 5)

\begin{tabular}{|c|c|c|c|}
\hline Study ID & & $\begin{array}{l}\text { Risk Ratio } \\
(95 \% \mathrm{Cl})\end{array}$ & $\begin{array}{l}\% \\
\text { Weight }\end{array}$ \\
\hline Tang et al. 2020 & $\stackrel{\leftrightarrow}{\longleftrightarrow}$ & $1.00(0.02,49.75)$ & 0.08 \\
\hline Gautret et al. 2020 & $\stackrel{\leftrightarrow}{\longleftrightarrow}$ & $1.89(0.08,43.75)$ & 0.06 \\
\hline Chen J et al. 2020 & $\stackrel{!}{\longleftrightarrow}$ & $1.00(0.02,47.38)$ & 0.07 \\
\hline Horby et al. 2020 & $\div$ & $1.07(0.97,1.19)$ & 99.64 \\
\hline Oriol et al. 2020 & $\stackrel{\leftrightarrow}{\longleftrightarrow}$ & $1.15(0.02,57.73)$ & 0.08 \\
\hline Chen C. P. et al. 2020 & $\stackrel{1}{\longleftrightarrow}$ & $0.59(0.01,28.03)$ & 0.08 \\
\hline Overall (I-squared $=0.0 \%)$ & 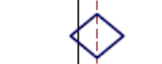 & $1.07(0.97,1.19)$ & 100.00 \\
\hline
\end{tabular}

Figure 5. Results from our updated meta-analysis assessing all-cause mortality.

\section{Secondary outcomes}

No significant differences between the groups in the risks of ICU transfer/mechanical ventilation, virological cure, and disease exacerbation. There was a significantly higher risk of adverse events in participants who received HCQ compared to those on standard care (Fig 6).

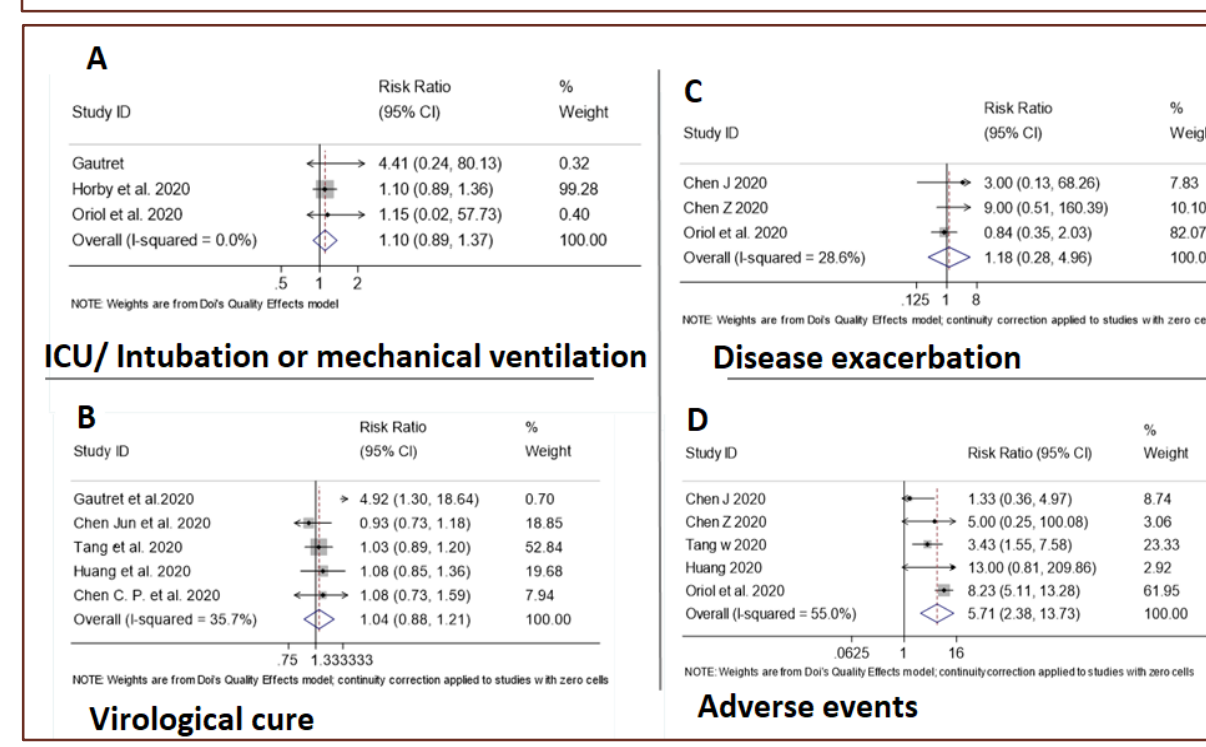

Figure 6. Updated meta-analysis of experimental studies on secondary outcomes.

\section{Conclusion}

There is conclusive evidence that CQ and HCQ, with or without Azithromycin are not effective in treating COVID-19 or its exacerbation.

\section{References}

